PROCEEDINGS OF THE

AMERICAN MATHEMATICAL SOCIETY

Volume 106, Number 1, May 1989

\title{
VIRTUALLY REGULAR COVERINGS
}

\author{
M. T. LOZANO AND C. SAFONT \\ (Communicated by Frederick R. Cohen)
}

\begin{abstract}
In this paper we introduce the concept of virtually regular covering, which include all regular branched coverings and also some irregular ones. We prove that, as in the case of regular coverings, some properties of the branching set reflect properties of the cover for virtually regular coverings of $S^{3}$, generalizing results of Kim-Tollefson, Lozano and Plotnick. As a consequence, we obtain that every virtually regular cover of $S^{3}$ branched along a fracturable prime and non split link is Haken.
\end{abstract}

\section{INTRODUCTION}

One way to study 3-manifolds is by representing them as covers of the sphere $S^{3}$ branched over links. It is known that some properties of the branching set reflect properties of the cover in the case of regular branched coverings. For instance, it was proved by Kim-Tollefson for double branched coverings [4], and by Plotnick for cyclic branched coverings [10] that the cover is irreducible if and only if the branching set is a prime and non split link. Gordon-Litherland proved [1] that if a regular branched cover is irreducible, then the branching set is prime and non split. Lozano showed [5] that a regular cover of $S^{3}$ branched over a fracturable prime link contains an incompressible surface of positive genus (fracturable link is defined in $\S 3$ ).

In $\S 1$, we introduce a new family of branched coverings, virtually regular coverings, which include all regular branched coverings and also some irregular ones.

In $\S 2$, we prove that a virtually regular cover of $S^{3}$ is irreducible if and only if the branching set is a prime and non split link, generalizing the results in [4, 10 and 1$]$.

In $\S 3$, we study virtually regular covers of $S^{3}$ branched over fracturable links, generalizing results of [5].

Received by the editors November 14, 1986 and, in revised form, May 2, 1988.

1980 Mathematics Subject Classification (1985 Revision). Primary 57M12; Secondary 57M25.

Key words and phrases. Branched coverings, incompressible surface, irreducible manifold, fracturable link.

The first author was supported by "Comisión Asesora de Investigación Cientificay Técnica." 
Through the paper we work with finite-fold coverings and in the PL-category. The definition of virtually regular covering is given for manifolds of any dimension $n \geq 2$. The branching set is supposed to be a submanifold of codimension 2. In $\S \S 2$ and 3 we deal with closed and orientable 3-manifolds. For the standard definitions of irreducible manifold, prime and split link, etc. (see [2 and 11]). We say that a manifold is reducible if it is not irreducible, i.e., if there exists some embedded 2-sphere which does not bound a 3-ball. A properly embedded surface $F$ in a 3-manifold is compressible if either $F$ is a 2-sphere bounding a 3-ball in $M$ or there exists a disc $D$ in $M$ such that $D \cap F=\partial D$ and $\partial D$ is not contractible in $F$. Otherwise, $F$ is incompressible.

\section{ViRTUALLY REgULAR COVERINGS}

Definition 1.1. ${ }^{1}$ A branched covering $p: M \rightarrow N$ is virtually regular if there exists an unbranched covering $u: M^{\prime} \rightarrow M$ such that $p \circ u: M^{\prime} \rightarrow N$ is regular.

Given a covering $p: M \rightarrow N$ branched over $Z$, with monodromy $\omega: \pi_{1}(N-Z) \rightarrow S_{n}$, the associated regular covering $q: X \rightarrow N$ is the branched covering determined by $\eta \circ \omega: \pi_{1}(N-Z) \rightarrow S_{\# \operatorname{Im} \omega}$, where $\eta$ is the regular representation of the group $\operatorname{Im} \omega$. Recall that $q=u \circ p$, where $u: X \rightarrow M$ is a regular (branched or unbranched) covering.

Theorem 1.2. $A$ branched covering $p: M \rightarrow N$ is virtually regular if and only if the branching index is constant along the fiber over each point of the branching set.

Proof. Suppose $p: M \rightarrow N$ is virtually regular. Let $u: M^{\prime} \rightarrow M$ be the unbranched covering such that $p \circ u: M^{\prime} \rightarrow N$ is regular. Consider a point $x$ of the branching set of $p$. Each point of $(p \circ u)^{-1}(x)$ has the same branching index $i_{x}$ because $p \cdot u$ is regular. As $u$ is unbranched, each point $y$ of $p^{-1}(x)$ has the same branching index as each point of $u^{-1}(y)$, hence $i_{x}$.

Conversely, suppose that the branching index of $p$ is constant along each fiber. Consider the associated regular covering $q=u \circ p: X \rightarrow N$. Let $x \in N$ be a point of the branching set. The branching index is constant along $q^{-1}(x)$, because $q$ is regular, and coincides with the branching index along $p^{-1}(x)$, because $\eta$ is injective. Therefore $u$ is unbranched.

Corollary 1.3. Let $p: M \rightarrow N$ be a branched covering with associated regular covering $q=u \circ p$. Then $p$ is virtually regular if and only if $u$ is unbranched.

Corollary 1.4. Let $M, N$ be 3-manifolds. A covering $p: M \rightarrow N$ branched over a link $L$ is virtually regular if and only if the monodromy map $\omega: \pi_{1}(N-L) \rightarrow S_{n}$ sends each meridian of $L_{i}$ to a product of cycles of the same length $h_{i}$, where $L_{i}$ is a component of $L$.

Virtually regular coverings of 3-manifolds branched over a link $L$ include, among others, the locally cyclic coverings, where the monodromy map sends

${ }^{1}$ The definition of virtually regular covering was suggested to us by J. M. Montesinos. 
each meridian of $L$ to a cycle of length $h$, if $h$ is the number of sheets of the covering; or, equivalently, $p$ maps $p^{-1}(L)$ homeomorphically onto $L$. Examples of irregular locally cyclic coverings of $S^{3}$ are given in [12]. There, Villarreal called locally cyclic manifold a manifold that can be represented as the cover of a locally cyclic covering of $S^{3}$, and investigated the problem of which 3-manifolds are locally cyclic. Here, we look at the natural generalization of that concept and that problem.

Definition 1.5. A 3-manifold $M$ is a virtually regular manifold if there exists some virtually regular covering $p: M \rightarrow S^{3}$ branched over a link.

It was shown by Villarreal in [13] that there are locally cyclic manifolds that are not cyclic (representable as cyclic branched covers of $S^{3}$ ).

Question 1. Is there any virtually regular manifold that is not regular (representable as regular branched cover of $S^{3}$ )?

Villarreal also found examples of manifolds which are not locally cyclic [14]. Question 2. Is every (closed, orientable) 3-manifold a virtually regular manifold?

\section{IRREDUCIBLE COVERS}

Let $r: M \rightarrow S^{3}$ be a regular covering of $S^{3}$ branched along a link $L$. Gordon and Litherland proved in [1] that $M$ irreducible implies $L$ prime. Actually, it is easy to prove that $M$ irreducible implies $L$ prime and nonsplit. The converse result ( $L$ prime and nonsplit implies $M$ irreducible) is proved for cyclic branched coverings (see [4 and 10]). The following theorem provides this converse result for any regular covering.

Theorem 2.1. Let $L$ be a link in $S^{3}$ and let $r: M \rightarrow S^{3}$ be a regular covering branched along $L$. If $M$ is reducible then $L$ is split or nonprime.

Proof. If $M$ is reducible, there exists a 2 -sphere $S$ which does not bound a 3-ball. Then $S$ is either nonseparating or $S$ decomposes $M$ as connected sum. In both cases there exists a nonempty family $\Gamma=\left\{S_{1}, \cdots, S_{k}\right\}$ of embedded disjoint 2-spheres in $M$ invariant under the action of the group $G$ of covering transformations, and such that $S_{i}$ does not bound a 3-ball for $i=1, \ldots, k$. (See $[8$, p. 480$]$ and $[10]$.

For each element $g$ of $G, g\left(S_{1}\right)=S_{1}$ or $g\left(S_{1}\right) \cap S_{1}=\varnothing$. Then $\left.r\right|_{S_{1}}: S_{1} \rightarrow F$, where $F$ is $r\left(S_{1}\right)$, is a (possibly branched) regular covering and $F$ is an embedded surface in $S^{3}$. Next we prove that $F$ is a 2-sphere which either intersects the link $L$ in two points or does not intersect $L$.

Let $g$ be the genus of $F, n$ the number of sheets of the covering $\left.r\right|_{S_{1}}$, $2 s$ the number of points of $F \cap L$, and $h$ the branch index of each point of $\left(\left.r\right|_{S_{1}}\right)^{-1}(F \cap L)$. The Hurwitz's formula [3] on Euler characteristic (note that $F$ is an orientable surface), gives

$$
2=\chi\left(S_{1}\right)=n(2-2 g-2 s)+2 s n / h .
$$


Two cases are possible:

(1) If $s=0$, then $1=n(1-g)$. This implies that $g=0$, i.e., $F$ is a 2-sphere which does not intersect $L$.

(2) If $s>1$, then $h=n h(1-g-s)+s n$. As $h$ divides $n$ and both of them are positive, this equation implies $n=h$ and $1=h(1-g-s)+s$. If $s>1$ or $g>0$, this yields the following contradiction, $s-1=h(s-1)+h g>s-1$. Therefore $s=1$ and $g=0$, i.e., $F$ is a 2-sphere and $F \cap L$ consists of two points.

In the first case, the link $L$ is split, because $F$ divides $S^{3}$ into two balls $B_{1}$ and $B_{2}$, each of them containing part of $L$. For if $B_{i} \cap L$ is empty, then $S_{1}$ bounds a 3-ball, which is not the case.

In the second case, the link $L$ is nonprime, because $F$ decomposes $L$ as connected sum of two nontrivial links $L_{1}$ and $L_{2}$. For if $L_{i}$ is trivial then $S_{1}$ bounds a 3-ball which is the cyclic covering of a ball branched along a spanning arc.

For an unbranched covering $u: X \rightarrow M$, where $X$ and $M$ are orientable 3-manifolds, it is a well known and trivial fact that if $X$ is irreducible, so is $M$. The converse was proved by Meeks-Simon-Yau using minimal surfaces [9, §7]. From this fact, Theorem 2.1 and [1], the following theorem follows.

Theorem 2.2. Let $p: M \rightarrow S^{3}$ be a covering branched along a link $L$, such that there exists a regular covering $r: N \rightarrow S^{3}$ branched along $L$ with the property that $N$ is an unbranched cover of $M$. Then $M$ is irreducible if and only if $L$ is prime and nonsplit.

Corollary 2.3. Let $p: M \rightarrow S^{3}$ be a virtually regular covering with branching set $L$. Then $M$ is irreducible if and only if $L$ is prime and nonsplit.

Remark. A direct proof of " $M$ irreducible implies $L$ prime" for locally cyclic coverings is given in [7].

Corollary 2.4 (See [10]). Let $L$ be a link in $S^{3}$. The following conditions are equivalent:

(a) $L$ is prime and nonsplit;

(b) all virtually regular covers of $S^{3}$ branched over $L$ are irreducible;

(c) one virtually regular cover of $S^{3}$ branched over $L$ is irreducible.

\section{FRACTURABLE LINKS AND INCOMPRESSIBLE SURFACES}

An arcbody [5] is a pair $\left(A, l_{A}\right)$, where $l_{A}$ is a 1 -manifold properly embedded in an orientable compact 3-manifold $A$ with boundary, with the following conditions:

(i) $A-l_{A}$ has incompressible boundary;

(ii) no component $\left(C, C \cap l_{A}\right)$ of $\left(A, l_{A}\right)$ is homeomorphic to $\left(D^{2} \times I\right.$, $\{0\} \times I)$ or $\left(D^{2} \times S^{1},\{0\} \times S^{1}\right)$, where $C$ is a connected component of $A$;

(iii) every sphere of $\partial A$ must intersect $l_{A}$. 
A cutting disc in $\left(A, l_{A}\right)$ is a properly embedded disc $D$ in $A$ intersecting $l_{A}$ transversally in exactly one interior point, and if $D$, together with a disc $E$ in $\partial A$, bounds a ball $D^{3} \subset A$, then $D^{3}$ does not intersect $l_{A}$ just in a spanning arc.

A link $\left(S^{3}, L\right)$ is fracturable [5] if there exists a compact closed surface $F$ embedded in $S^{3}$ dividing $\left(S^{3}, L\right)$ in two arcbodies. Then $F$ gives a fracture of $\left(S^{3}, L\right)$. A fracture is minimal if $F$ is connected and there are no cutting discs in any of the two arcbodies of the fracture.

It is proved in [5] that every regular cover of $S^{3}$ branched over a fracturable and prime link contains an incompressible surface of positive genus. Here we generalize this result to virtually regular coverings.

Lemma 3.1. Let $u: X \rightarrow M$ be an unbranched covering where $X$ and $M$ are 3-manifolds. Let $T$ be an orientable embedded closed surface in $M$ such that a component $R$ of $u^{-1}(T)$ is an incompressible surface in $X$ of positive genus. Then $T$ is incompressible and has positive genus.

Proof. The restriction map $\left.u\right|_{R}: R \rightarrow T$ is an unbranched covering, so the genus of $T$ is positive.

To prove that $T$ is incompressible, consider an embedded disc $D$ in $M$ such that $D \cap T=\partial D$. The preimage $u^{-1}(D)$ is a disjoint union of discs, and the boundary of one of them, say $D_{1}$, lies on $R$. Then $\partial D_{1}$ bounds a disc $E$ in $R$ because $R$ is incompressible. It follows that $\partial D$ is a separating curve in $T$. Let $T_{1}$ and $T_{2}$ be the two parts of $T$ separated by $\partial D$. Then $u^{-1}(T)=u^{-1}\left(T_{1}\right) \bigcup_{u^{-1}(\partial D)} u^{-1}\left(T_{2}\right)$ and $\left.u\right|_{E}: E \rightarrow T_{i}$ is a covering (for $i=$ 1 or 2) which is a homeomorphism on the boundary. This implies that $T_{i} \quad(i=$ 1 or 2 ) is a disc. Therefore $T$ is incompressible.

Theorem 3.2. Let $\left(S^{3}, L\right)$ be a fracturable prime link and let $p: M \rightarrow S^{3}$ be a virtually regular covering branched over $L$. Then $M$ contains an incompressible surface of positive genus.

Proof. Let $F$ be an embedded surface in $S^{3}$ giving a minimal fracture of $\left(S^{3}, L\right)$. [5, Proposition 2.1] assures that such a surface exists. We will prove that every component of $p^{-1}(F)$ is an incompressible surface in $M$ of positive genus. Let $T$ be one of these components. Consider the unbranched covering $u: X \rightarrow M$ such that $r=p \circ u: X \rightarrow S^{3}$ is regular. Then $u^{-1}(T)$ is a disjoint union of components of $r^{-1}(F)$. Since each of these components is an incompressible surface of positive genus [5, Theorem 3.1], $T$ is incompressible and has positive genus by Lemma 3.1.

The following Lemma 3.3 is a converse of [ 5 Theorem 3.1]. The analogous result for regular coverings branched over 3-braids is contained in [6, Corollary 3.8]. Theorem 3.5 is the generalization to virtually regular coverings. 
Lemma 3.3. Let $L$ be a nonsplit link in $S^{3}$, and let $r: N \rightarrow S^{3}$ be a regular covering branched over $L$. If there exists a closed embedded connected surface $F$ in $S^{3}$ cutting $L$ transversally, and such that a component $R$ of $r^{-1}(F)$ is incompressible, then $F$ gives a fracture of $\left(S^{3}, L\right)$. Moreover, if $L$ is prime then this fracture is minimal.

Proof. We will prove that $(A, A \cap L)$ and $(B, B \cap L)$ are arcbodies, where $A$ and $B$ are the two parts of $S^{3}$ bounded by $F$.

(i) The inclusion map $i_{A}: F-L \hookrightarrow A-A \cap L$ is monic on $\pi_{1}$ : Let $\alpha$ be a loop in $F-L$ such that there exists a disc $D$ properly embedded in $A-A \cap L$ whose boundary is $\alpha$. As $D$ does not intersect $L, r^{-1}(D)$ is the union of $n$ pairwise disjoint discs $\Delta_{i}(i=1, \ldots, n)$, where $n$ is the number of sheets of the covering $r$. For some $i, \partial \Delta_{i}$ is contained in $R$. Since $R$ is incompressible there exists a disc $E_{i}$ contained in $R$ such that $\partial E_{i}=\partial \Delta_{i}$. For $j=1, \ldots, n$, there exists a covering transformation $g_{j}$ such that $g_{j}\left(E_{i}\right)$ is a disc with boundary $g_{j}\left(\partial \Delta_{i}\right)=\partial \Delta_{j}$. Call $g_{j}\left(E_{i}\right)=E_{j}$. The set $\left\{E_{j} \mid j=1, \ldots, n\right\}$ is a family of discs embedded in the surface $r^{-1}(F)$ and they have disjoint boundaries. This means that every component of $\bigcup_{j=1}^{n} E_{j}$ is a disc. Let $C$ be one of these components, and consider the restriction map $\left.r\right|_{C}: C \rightarrow r(C)$ which is an $m$ to 1 regular (branched) covering for some $m$. Using the Hurwitz formula on Euler characteristic [3], we have

$$
1=\chi(C)=m \chi(r(C))-a(m-l)
$$

where $a$ is the number of points of $r(C) \cap L$, and $l$ is the number of points in the preimage by $\left.r\right|_{C}$ of any point of $r(C) \cap L$. Then $l$ divides $m$, and $l \neq m$. From (1) we obtain

$$
\chi(r(C))=\frac{1+a(m-l)}{m}>0 .
$$

The only possible value of $a$ is zero. For if $a>1, \chi(r(C))>1$, which is impossible. And if $a=1, D \cup r(C)$ is a 2-sphere in $S^{3}$ intersecting $L$ in one point, which is also impossible. Thus, $a=0$ and $\chi(r(C))=1$, and $\left.r\right|_{C}$ is a homeomorphism. Therefore $r(C)$ is a disc contained in $F-L$ with boundary $\alpha$. Hence $\alpha$ is contractible in $F-L$ and the map $i_{A}$ is monic.

(ii) Since $r$ is a regular branched covering, every component of $r^{-1}(F)$ is the image of $R$ by some covering transformation. As $R$ is incompressible, every component of $r^{-1}(F)$ is also incompressible. It follows that $(A, A \cap L)$ is not homeomorphic neither to $\left(D^{2} \times I,\{0\} \times I\right)$ nor to $\left(D^{2} \times S^{1},\{0\} \times S^{1}\right)$.

(iii) There are not 2-spheres in $\partial A-L=F-L$. For if $F$ is a 2 -sphere disjoint of $L, R$ is a 2-sphere which does not bound a 3-ball. Then $L$ is split.

Therefore $(A, A \cap L)$ and $(B, B \cap L)$ are arcbodies, which proves the first part of the theorem.

To prove that if $L$ is prime the fracture $\left(S^{3}, L\right)$ is minimal, we will prove that there are not cutting-discs in $(A, A \cap L)$ and $(B, B \cap L)$. Suppose that $D$ 
is a properly embedded disc in $A$ or $B$ such that $D$ intersects transversally $L$ in one interior point of $D$. Let $E$ be a component of $r^{-1}(D)$ and $\left.r\right|_{E}: E \rightarrow D$ is the cyclic covering ( $h$ to 1 ) of $D$ branched along the point $D \cap L$. The boundary of $E$ lies in the incompressible surface $r^{-1}(F)$. Thus $\partial E$ bounds a disc $E^{\prime}$ in $r^{-1}(F)$. A similar argument of the above one, using Hurwitz's formula, proves that $E^{\prime}$ projects to a disc $C$ intersecting transversally $L$ in one point, and $C \cup D$ is a 2-sphere cutting $L$ in two points. Since $L$ is prime, one of the two balls bounded by $C \cup D$ intersects $L$ in one spanning arc. Therefore $D$ is not a cutting disc.

Lemma 3.4. Let $u: X \rightarrow M$ be an unbranched covering, where $X$ and $M$ are orientable 3-manifolds. Let $T$ be an orientable incompressible embedded surface in $M$ of positive genus, then each component of $u^{-1}(T)$ is an incompressible surface in $X$ of positive genus.

Proof. Let $R$ be a connected component of $u^{-1}(T)$. The restriction map $\left.u\right|_{R}: R \rightarrow T$ is a covering, so the genus of $R$ is greater than or equal to the genus of $T$, hence positive.

Since $T$ is a 2-sided incompressible surface in $M, i_{T \#}$ is a monomorphism [2, Corollary 6.2], where $i_{T \#}: \pi_{1}\left(T, u\left(x_{0}\right)\right) \rightarrow \pi_{1}\left(M, u\left(x_{0}\right)\right), x_{0} \in R$, is induced by the inclusion $i_{T}: T \rightarrow M$. We know that $\left.u\right|_{R \#}$ and $u_{\#}$, and hence $i_{T \#} \circ$ $\left.u\right|_{R^{\#}}=u_{\#} \circ i_{R \#}$ are monomorphisms, where $i_{R \#}: \pi_{1}\left(R, x_{0}\right) \rightarrow \pi_{1}\left(X, x_{0}\right)$ is induced by the inclusion $i_{R}: R \rightarrow X$. Therefore $i_{R \#}$ is also a monomorphism, and thus $R$ is incompressible in $X$.

Theorem 3.5. Let $p: M \rightarrow S^{3}$ be a virtually regular covering branched along a nonsplit link $L$. If there exists an embedded connected closed surface $F$ in $S^{3}$ cutting $L$ transversally, and such that a component $R$ of $p^{-1}(F)$ is incompressible, then $\left(S^{3}, L\right)$ is fracturable with fracture $F$. And if $L$ is prime, then $F$ is a minimal fracture of $\left(S^{3}, L\right)$.

Proof. Consider the unbranched covering $u: X \rightarrow M$, such that $p \circ u: X \rightarrow S^{3}$ is regular. By Lemma 3.4, every component of $u^{-1}(R)$ is an incompressible surface in $X$, and it is also a component of $r^{-1}(F)$. Then by Theorem 3.3, it follows the thesis.

Remark. A direct proof of Theorems 3.2 and 3.5 for locally cyclic coverings is given in [7].

Corollary 3.6. All virtually regular covers of $S^{3}$ branched over a fracturable and nonsplit link are Haken manifolds.

\section{REFERENCES}

1. C. McA. Gordon and R. A. Litherland, Incompressible surfaces in branched coverings, Symposium on the Smith Conjecture (Bass and Morgan, eds.) Academic Press, New York, 1984.

2. J. Hempel, 3-manifolds, Ann. of Math. Siud., no. 86, Princeton Univ. Press, Princeton, N.J., 1976. 
3. A. Hurwitz, Über Riemann'sche Flächen mit gegebenen verzweigungspunkten, Math. Ann. 39 (1891), 1-60.

4. P. K. Kim and J. L. Tollefson, Spliting the PL involutions of nonprime 3-manifolds, Michigan Math. J. 27 (1980), 259-274.

5. M. T. Lozano, Arcbodies, Math. Proc. Cambridge Philos. Soc. 94 (1983), 253-260.

6. M. T. Lozano and J. Przytycki, Incompressible surfaces in the exterior of a closed 3 braid. I, Math. Proc. Cambridge Philos. Soc. 98 (1985), 275-299.

7. M. T. Lozano and C. Safont, Sobre cubiertas localmente ciclicas, Contribuciones Matematicas. Estudios en honor del Profesor Botella. U. C. de Madrid (1986), 169-175.

8. W. H. Meeks III and S. T. Yau, Topology of three dimensional manifolds and the embedding problems in minimal surface theory, Ann. of Math. (2) 112 (1980), 441-484.

9. W. Meeks III, L. Simon and S. T. Yau, Embedded minimal surfaces, exotic spheres, and manifolds with positive Ricci curvature, Ann. of Math. 116 (1982), 621-659.

10. S. P. Plotnick, Finite group actions and nonseparating 2-spheres, Proc. Amer. Math. Soc. 90 (1984), 430-432.

11. D. Rolfsen, Knots and links, Publish or Perish, Inc., 1976.

12. L. Villarreal, Recubridores localmente ciclicos, Tesis doctoral Univ. Complutense de Madrid (1984).

13. __ Una variedad localmente cíclica que no es cíclica, Actas VII Congreso de Matemáticos de Expresión Latina, vol 1, 1986, pp. 525-528.

14. __ Los recubridores localmente ciclicos no son universales (1985), preprint.

Departamento de Geometría y Topología, Facultad de Ciencias, Universidad de Zaragoza, SPAIN 